\title{
Combining drugs to optimize the therapy of hypertension: experimental evidence derived from animal models
}

\author{
Danielle A Guimaraes and Jose E Tanus-Santos
}

Hypertension Research (2015) 38, 457-458; doi:10.1038/hr.2015.52; published online 2 April 2015

$\mathrm{H}$ ypertension is widely known to promote renal disease and this is associated with complex pathophysiological mechanisms including enhanced renal sympathetic activity and the hyperactivation of the renin-angiotensin-aldosterone system (RAAS). Increased sympathetic nervous system stimulation activates renin release, sodium reabsorption, vasoconstriction, and decreased renal blood flow. ${ }^{1}$ Abnormal activation of the RAAS and increased renin secretion lead to increased angiotensin II production, which in turn activates multiple intracellular signaling pathways (Figure 1) that result in increased blood pressure and a variety of functional and structural alterations of the cardiovascular system. $^{2}$

The study by Tiradentes et al. ${ }^{3}$ used the two-kidney one-clip (2K1C) hypertension model, which resembles human severe hypertension and is clearly dependent on renin-angiotensin-aldosterone system (RAAS) activation. $^{2}$ The authors took advantage of a very interesting physiological approach to show that the combination of aliskiren (a renin inhibitor) and L-arginine (a substrate for nitric oxide, NO, synthesis) effectively lowers blood pressure in 2K1C hypertensive rats in association with improved renal function and reduced renal sympathetic nerve activity (RNSA). ${ }^{3}$ Although a clear mechanistic link between reduced RNSA and drug effects is not provided in this study, the authors concluded that RNSA contributes to renovascular hypertension, and that combining aliskiren and L-arginine reduces RNSA,

DA Guimaraes and JE Tanus-Santos are at Department of Pharmacology, Ribeirao Preto Medical School, University of Sao Paulo, Sao Paulo, Brazil

E-mail: tanus@fmrp.usp.br or tanussantos@yahoo.com improves renal dysfunction and lowers blood pressure. ${ }^{3}$ This study provides experimental evidence that may contribute to the improved understanding of hypertensive mechanisms and therapeutic approaches.

Aliskiren has recently been approved for the therapy of hypertension with the idea that inhibiting renin activity could provide substantial additional protection, at least to some patients, as compared with other traditional antihypertensive drugs. Indeed, direct renin inhibitors, by interfering with the initial steps in the RAAS cascade (conversion of angiotensinogen to angiotensin I), could provide a complete blockade of the RAAS. ${ }^{4}$ As monoterapy, aliskiren 150,300 and $600 \mathrm{mg}$ once a day lowered blood pressure in mild-to-moderate hypertensive patients and

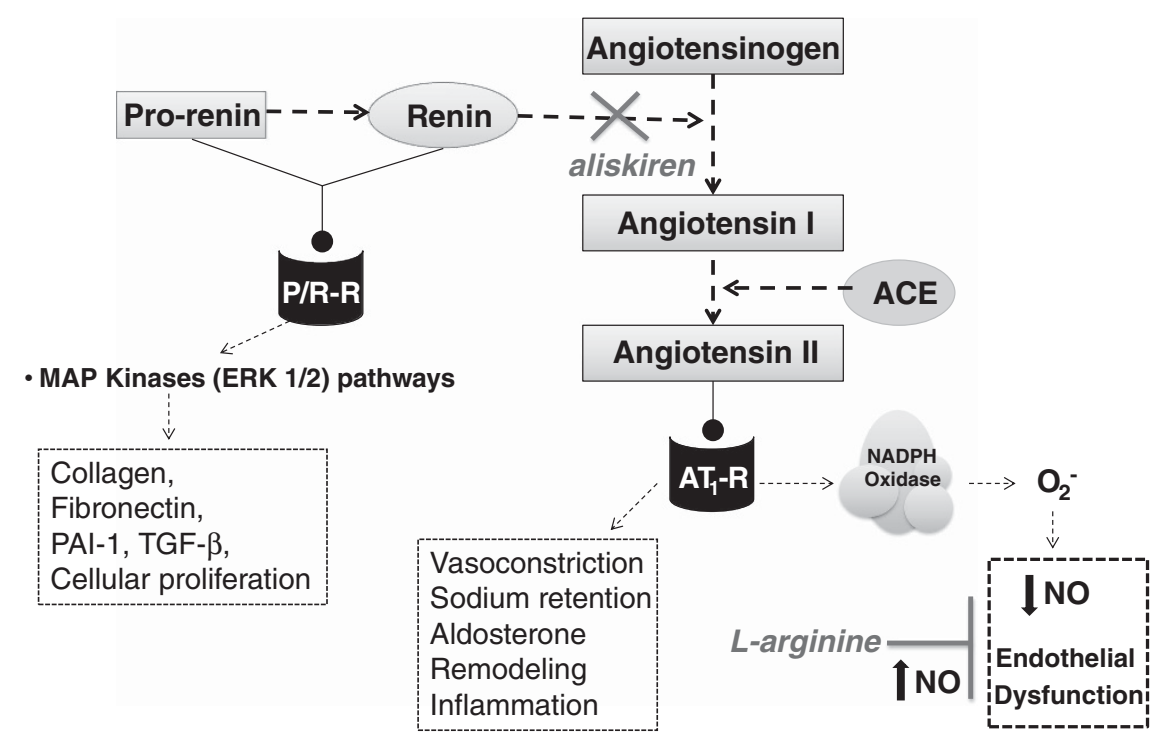

Figure 1 Signaling pathways involved in the pathophysiology of hypertension. Activation of the renin angiontensin aldosterone system (RAAS) is commonly found in different animal models of hypertension. Increased angiotensin II concentrations increase blood pressure by activating hypertensive mechanisms and promotes cardiovascular inflammation and remodeling. Although aliskiren inhibits renin activity, thus decreasing the overall activity of the RAAS and angiotensin II concentrations, both renin and prorenin can bind to receptors and activate a variety of profibrotic pathways, therefore limiting the responses to aliskiren. Angiontensin II activation of $A T_{1} R$ also increases NADPH oxidase activity, a major contributor to superoxide formation in hypertension. L-arginine is a substrate for nitric oxide (NO) formation, and therefore can replenish NO bioavailability, which is impaired by increased oxidative stress in hypertension. ACE, angiotensin converting enzyme; $A T_{1} R$, angiotensin II type 1 receptors; $\mathrm{NADPH}$, nicotinamide adenine dinucleotide phosphate oxidase; P/R-R, prorenin and renin receptors. A full color version of this figure is available at Hypertension Research online. 
this drug is apparently as effective as angiotensin receptor blockers. ${ }^{5}$ However, althouigh the inhibition of RAAS is often used in the therapy of hypertension and its complications, many patients will require combinations of drugs to effectively control blood pressure, ${ }^{4}$ and therefore examining different combinations may be very helpful.

To improve the effects of aliskiren, Tiradentes et al. combined this renin inhibitor with L-arginine, a substrate to increase NO bioavailability. The rationale for this approach is very consistent with a number of previous studies showing that NO deficiency contributes to hypertension and that therapeutic approaches designed to replenish NO deficiency may be very effective in lowering blood pressure. ${ }^{6}$ In fact, it is clear that the activation of angiotensin II type 1 receptors $\left(\mathrm{AT}_{1} \mathrm{~s}\right)$ is associated with increased reactive oxygen species formation (particularly superoxide) as a result of angiotensin II-induced upregulation of nicotinamide adenine dinucleotide phosphate oxidase oxidase activity (Figure 1), particularly in the $2 \mathrm{~K} 1 \mathrm{C}$ hypertension model. ${ }^{7}$ The activation of this mechanism in hypertension decreases $\mathrm{NO}$ activity and causes endothelial dysfunction.

Therefore, enhancing NO formation with L-arginine treatment may provide an additional, protective mechanism to counteract deleterious alterations found in the $2 \mathrm{~K} 1 \mathrm{C}$ hypertension model, as shown by Tiradentes et al., possibly by improving a vasodilatory mediator (NO) to improve renal vasoconstriction triggered by elevated angiotensin II levels.

Interestingly, Tiradentes et $a l^{3}$ showed that 3 weeks of treatment with combined L-arginine or aliskiren exerted major antihypertensive effects, whereas treatment with L-arginine had only minor effects, and aliskiren alone had no effects. This observation may suggest that improving NO bioavailability may be more relevant that inhibiting renin activity in the $2 \mathrm{~K} 1 \mathrm{C}$ hypertension model. To further examine the role of $\mathrm{NO}$, the authors tried to explore which isoforms of $\mathrm{NO}$ synthases are possibly involved in the pathophysiological alterations of renovascular hypertension. Although the authors advocate a possible protective effect of increased inducible NO synthase (iNOS) in this model, particularly to the ischemic kidney, ${ }^{3}$ experimental findings in another animal model of severe hypertension in pregnancy contrasts with this suggestion. ${ }^{8}$ Indeed, it is possible that abnormal amounts of $\mathrm{NO}$ produced by iNOS enhances peroxinitrite levels, particularly in a context of increased oxidative stress, which is associated with increased superoxide production, thus leading to nitrosative stress and cardiovascular dysfunction. ${ }^{9}$ Clearly, this is a very complex issue, which would be better explored with by carrying out experiments with knockout mice or by using selective NO synthase inhibitors.

The lack of significant effects after therapy with aliskiren alone, as reported by Tiradentes et al., is not surprising. A previous study by our group compared the effects of monotherapy with the $\mathrm{AT}_{1}$ receptor antagonist losartan with those found after monoterapy with aliskiren, or the combination of both the drugs in $2 \mathrm{~K} 1 \mathrm{C}$ hypertensive rats. ${ }^{10}$ In agreement with the findings reported by Tiradentes et al., ${ }^{3}$ aliskiren alone did not prevent the vascular remodeling and profibrotic alterations found in $2 \mathrm{~K} 1 \mathrm{C}$ hypertension, ${ }^{10}$ even though this drug significantly reduced the plasma renin activity. In contrast, losartan or the combination of drugs prevented most biochemical and structural vascular alterations associated with $2 \mathrm{~K} 1 \mathrm{C}$ hypertension. ${ }^{10}$ Together, these results suggest that critical mechanisms not significantly affected by renin inhibition may exert major effects. For example, it is possible that the binding of prorenin and renin to their receptors triggers a series of intracellular signaling pathways including mitogenactivated protein kinases that contribute to the alterations of hypertension ${ }^{10}$ (Figure 1). From the clinical standpoint, a recently published meta-analysis carried out to compare the antihypertensive effects and tolerability of aliskiren with other antihypertensives showed overall similar effects of aliskiren as compared with other drugs, particularly when aliskiren was compared with angiontensin receptor blockers. ${ }^{11}$

Given the complexity of pathophysiological mechanisms interacting to increase blood pressure and to remodel the cardiovascular system in hypertension, it is highly probable that combinations of drugs will always be required to manage hypertensive patients. Future studies assessing the antihypertensive effects of various combinations of drugs in different animal models may help to define improved therapies.

\section{CONFLICT OF INTEREST}

The authors declare no conflict of interest.

\section{ACKNOWLEDGEMENTS}

We are supported by Conselho Nacional de Desenvolvimento Científico e Tecnológico (CNPq-Brazil), Coordenação de Aperfeiçoamento de Pessoal de Nível Superior (CAPES-Brazil) and Fundação de Amparo a Pesquisa do Estado de São Paulo (FAPESP-Brazil).

1 Sorota S. The sympathetic nervous system as a target for the treatment of hypertension and cardiometabolic diseases. J Cardiovasc Pharmacol 2014; 63: 466-476.

2 Ceron CS, Rizzi E, Guimaraes DA, Martins-Oliveira A Cau SB, Ramos J, Gerlach RF, Tanus-Santos JE. Time course involvement of matrix metalloproteinases in the vascular alterations of renovascular hypertension. Matrix Biol 2012; 31: 261-270.

3 Tiradentes RV, Santuzzi CH, Claudio ERG, Mengal V, Silva NF, Neto HAF, Bissoli NS, Abreu GR, Gouvea SA Combined Aliskiren and L-arginine treatment reverses renovascular hypertension in an animal model. Hypertens Res 2015; 38: 471-477.

4 Rashid HU, Mende C. The role of direct renin inhibition in clinical practice: focus on combination therapy. $A m \mathrm{~J}$ Cardiovasc Drugs 2011; 11: 303-315.

5 Gradman AH, Schmieder RE, Lins RL, Nussberger J, Chiang $\mathrm{Y}$, Bedigian MP. Aliskiren, a novel orally effective renin inhibitor, provides dose-dependent antihypertensive efficacy and placebo-like tolerability in hypertensive patients. Circulation 2005; 111 1012-1018.

6 Montenegro MF, Pinheiro LC, Amaral JH, Marcal DM Palei AC, Costa-Filho AJ, Tanus-Santos JE. Antihypertensive and antioxidant effects of a single daily dose of sodium nitrite in a model of renovascular hypertension. Naunyn Schmiedebergs Arch Pharmacol 2012; 385: 509-517.

7 Montenegro MF, Amaral JH, Pinheiro LC, Sakamoto EK, Ferreira GC, Reis RI, Marçal DMO, Pereira RP, Tanus-Santos JE. Sodium nitrite downregulates vascular NADPH oxidase and exerts antihypertensive effects in hypertension. Free Radic Biol Med 2011; 51: 144-152.

8 Amaral LM, Pinheiro LC, Guimaraes DA, Palei AC Sertorio JT, Portella RL, Tanus-Santos JE. Antihypertensive effects of inducible nitric oxide synthase inhibition in experimental pre-eclampsia. J Cell Mol Med 2013; 17: 1300-1307.

9 Oliveira-Paula GH, Lacchini R, Tanus-Santos JE. Inducible nitric oxide synthase as a possible target in hypertension. Curr Drug Targets 2014; 15: 164-174.

10 Martins-Oliveira A, Castro MM, Oliveira DM, Rizzi E, Ceron CS, Guimaraes D, Reis RI, Costa-Neto CM, Casarini DE, Ribeiro AA, Gerlach RF, Tanus-Santos JE. Contrasting effects of aliskiren versus losartan on hypertensive vascular remodeling. Int J Cardiol 2013; 167: 1199-1205.

11 Chen Y, Meng L, Shao H, Yu F. Aliskiren vs. other antihypertensive drugs in the treatment of hypertension a meta-analysis. Hypertens Res 2013; 36: 252-261. 